\title{
Multi system inflammatory syndrome in children and adolescents temporally related to COVID-19
}

\author{
Mrs Suman M Pawar \\ MSc (N), Professor, College of Nursing, Wanless Hospital, Miraj. \\ Corresponding Email: smpawar79@rediffmail.com
}

Background: The pandemic of COVID -19 since its eruption from Wuhan, till date have rapidly captured the whole world. As of May 2020, 4 million confirmed cases of COVID-19, 285000 deaths have been reported to WHO. Children have borne a minimal medical burden to the health care system. Children and the teenagers have been spared during this global COVID pandemic. However there have been very few cases of children seriously affected by COVID -19 . Epidemiological data from many countries show that children younger than 18 years have made up only $1 \cdot 7 \%$ of national cases in the USA, ${ }^{1} 1 \%$ of cases in the Netherlands, ${ }^{2}$ and $2 \cdot 0 \%$ of a large observational cohort in the UK. These proportions are low showing susceptibility of COVID -19 among children than in adults. But children may have similar high proportion rates of asymptomatic disease which is unclear. Recently some cases have been reported from Europe and North America describing clusters of children and adolescents with a multisystem inflammatory condition like some features similar to those of Kawasaki disease and toxic shock syndrome. All of them requiring admission to intensive care units. This is a new syndrome relating to COVID -19 positive children. Much is not know about the full spectrum of the disease. Thus it is essential to characterize this syndrome and its risk factors. We also should get to understand its causality and describe the treatment interventions. There is therefore an urgent need for collection of standardized data describing clinical presentations, severity, outcomes, and epidemiology.

Keyword: Multi system inflammatory syndrome, children, adolescents,covid-19

\section{What is Multisystem inflammatory Syndrome-Children (MIS-C)?}

It is a rare, mysterious condition where different body parts can become inflamed including the heart, lungs, kidneys, brain, skin, eyes or GI organs. It causes inflammation of the blood vessels throughout the body. The inflammation can limit blood flow, damaging the heart, kidneys and other organs.
The cause is yet not know. The incidences seen that the syndrome can develop within four weeks of exposure to the new coronavirus. Its seems to affect children between 2 years to 15 years. The cases are not been reported in babies. This is a newly identified condition and much more research is needed to understand it. The prognosis can be serious even deadly...but can get better with medical care.

Symptoms of MIS-C 
$\checkmark$ fever (24hours)

$\checkmark \quad$ abdominal (belly) pain

$\checkmark \quad$ vomiting

$\checkmark$ diarrhoea

$\checkmark \quad$ neck pain

$\checkmark \quad$ rash

$\checkmark \quad$ blood shot eyes

$\checkmark$ feeling extra tired

$\checkmark$ Swollen hands or feet

Be aware that not all children will have all the same symptoms.

\section{Emergency-Warning signs of MIS-C}

Severe Symptoms of MIS-C

- Trouble breathing,

- Pain pressure in chest,

- Inability to awake or stay awake.

- Bluish lips or face

- Severe abdominal pain

\section{How will the doctors care for your child?}

Doctors may do certain tests to look for inflammation or other signs of disease.

These tests might include:

- Blood tests

- Chest x-ray

- Heart ultrasound (echocardiogram)

- Abdominal ultrasound

\section{Treatment of MIS-C}

MIS-C is treatable if it is detected

* Most ill children will need hospitalization.
Some will need to be treated in the pediatric intensive care unit (ICU).

Treatment usually involves different types of therapies that target the immune system and reduce inflammation.

Doctors can use medicines such as

$>$ intravenous immunoglobulin,

$>$ steroids and

$>$ other anti-inflammatory drugs

The goal is to reduce the inflammation and protect the heart, kidneys and other organs from lasting damage.

MIS-C and COVID-19 :What Is the Connection?

MIS-C was first identified in April 2020, at children's hospitals in the United States and the United Kingdom. The condition has also been called pediatric inflammatory multisystem syndrome (PIMS). Dr Kwang Sik Kim, M.D a specialist in pediatric infectious disease and Director at Johns Hopkins Children's Center was instrumental in providing information about MIS-C. He said that many children with MIS-C had the virus that causes COVID-19.It has also been observed that the syndrome may be due to the body's immune response to the presence of the SARS-CoV-2 coronavirus.

What we don't know about MIS-C? 
The U.S. Centers for Disease Control and Prevention (CDC) recently recognized this new syndrome in children and teens ages 2-15years.... its associated with the coronavirus that causes COVID-19. $\mathrm{CDC}$ is still learning about MIS-C and investigating how it affects children. We don't know why some children have got sick with MIS-C and others have not. We also do not know if children with certain health conditions are more likely to get MIS-C. These are among the many questions CDC is working to try to understand. All CDC recommendations are based on the best data and science available at the time, and we will update them as we learn more.

\section{What CDC Is Doing to learn About Multisystem Inflammatory Syndrome in Children (MIS-C).}

The team is working for US, international scientists and the health care providers to learn more about new syndrome.

CDC has a team dedicated to investigate, gather, communicate, information quickly to health care providers, parents.

They are learning about

○ how often it happens and

○ who is likely to get it,

○ creating a system to track cases, and

o providing guidance to parents and healthcare providers.

\section{Some of the CDC Activities:}

\section{CDC are working with partners}

- Collaborating with public health agencies to share information, knowledge about cases of MIS-C in other countries.

- Providing local support to investigation into MIS-C cases.

- Understanding the appearance of disease in children - outcomes of MIS-C cases.

- Identifying additional cases of MIS-C associated with COVID-19, determine risk factors for MIS-C

- Asking clinical research and surveillance data networks at children's hospitals

\section{Preparing Healthcare Providers and Health Departments}

- Released a Health Advisory on May 14, 2020, through the Health Alert Network.

- Keeping Alerted the health care providers

- Issued the case definition ......developed with the Council of State, Tribal, and Territorial Epidemiologists

- Hosted a webinar with researchers and clinicians who have treated patients with MIS-C. 
- Set up a method to report cases of MISC for state and local health departments

\section{Keeping Parents and Partners Informed}

- Communicating information about what we know, what we don't know, and what we are doing to learn more to support healthcare providers, as well as parents and caregivers.

- Conducting- partner outreach activities and educational efforts to increase provider awareness of MISC.

- Collaborating with other federal agencies, clinical, and professional societies.

How to help prevent your child from getting MIS-C

Follow the guidelines of the U.S. Centers for Disease Control and Prevention:

- Keep hands clean. Wash hands often with soap and water for at least 20 seconds. If soap and water aren't available, use a hand sanitizer containing at least $60 \%$ alcohol.

- Avoid people who are sick. In particular, avoid people who are coughing, sneezing or showing other signs and symptoms that indicate they might be sick and contagious.

- Practice social distancing. This means that you and your child should stay at least 6 feet ( 2 meters) from other people when outside of your home.

- Wear cloth face masks in public settings. When it's difficult to practice social distancing, both you and your child - if he or she is age 2 years or older - should wear face masks that cover the nose and mouth.

- Clean and disinfect high-touch surfaces every day. This includes areas of your home such as doorknobs, light switches, remotes, handles, countertops, tables, chairs, desks, keyboards, faucets, sinks and toilets.

- Wash clothing and other items as needed. Follow manufacturers' instructions, using the warmest appropriate water setting on your washing machine. Remember to include washable plush toys.

WHO developed preliminary case definition and case reports form for multisystem inflammatory disorder in children

Preliminary case definitions-0-19yrs

With fever $=3$ days or more and 2 of the following 
Rash /bilateral purulent conjuctivitis,or muco cutaneous inflammatory signs(hands, feet).

$>$ Hypotension/shock

$>$ Features of myocardial dysfunctions(pericarditis, valvulitis, coronary abnormality ECHO, elevated troponin)

$>$ Elevated coagulopathy (PT,PTT,elevated d-Dimers)

$>$ Elevated markers of inflammtion such as ESR, C-reactive protein, or procalcitonin.

The preliminary case definition reflects the clinical and laboratory features observed in children reported to date, and serves to identify suspected or confirmed cases both for the purpose of providing treatment and for provisional reporting and surveillance. The case definition will be revised as more data become available.

A Case report: Hyperinflammatory shock in children during COVID-19 pandemic (Lancet MAY 2020)

In 10 days period between mid April 2020 was noted an unprecedented cluster of eight children with hyperinflammatory shock. The demographic variables showing six of the children were of Afro-Caribbean descent, and five of the children were boys.
All children except one were above the 75th percentile .Four children had known family exposure to (COVID-19). Clinical presentations in all the children were similar, with unrelenting fever $\left(38-40^{\circ} \mathrm{C}\right)$, variable rash, conjunctivitis, peripheral oedema, and generalised extremity pain with significant gastrointestinal symptoms. All progressed to warm, vasoplegic shock, refractory to volume resuscitation and eventually requiring noradrenaline and milrinone for haemodynamic support. Most of the children had no significant respiratory involvement, although seven of the children required mechanical ventilation for cardiovascular stabilisation. Other notable features (besides persistent fever and rash) included development of small pleural, pericardial, and ascitic effusions, suggesting of a diffuse inflammatory process. All children tested negative for severe acute respiratory syndrome coronavirus 2 (SARSCoV-2) on broncho-alveolar lavage or nasopharyngeal aspirates. Despite being critically unwell, with laboratory evidence of infection or inflammation ${ }^{3}$ including elevated concentrations of C-reactive protein, procalcitonin, ferritin, triglycerides, and D-dimers, no pathological organism was identified in seven of the children. Baseline electrocardiograms were non-specific; 
however, a common echocardiographic finding was echo-bright coronary vessels (which progressed to giant coronary aneurysm in one patient within a week of discharge from paediatric intensive care . One child developed arrhythmia with refractory shock, requiring extracorporeal life support, and died from a large cerebrovascular infarct. The myocardial involvement ${ }^{2}$ in this syndrome is evidenced by very elevated cardiac enzymes during the course of illness. All children were given intravenous immunoglobulin $(2 \mathrm{~g} / \mathrm{kg})$ in the first $24 \mathrm{~h}$, and antibiotic cover including ceftriaxone and clindamycin. Subsequently, six children have been given $50 \mathrm{mg} / \mathrm{kg}$ aspirin. All of the children were discharged from PICU after 4-6 days. Since discharge, two of the children have tested positive for SARS-CoV-2 (including the child who died, in whom SARS-CoV-2 was detected post mortem). These clinical picture represents a new phenomenon affecting previously asymptomatic children with SARS-CoV-2 infection manifesting as a hyperinflammatory syndrome with multiorgan involvement similar to Kawasaki disease shock syndrome. The multifaceted nature of the disease course underlines the need for multispecialty input (intensive care, cardiology, infectious diseases, immunology, and rheumatology). The intention of this Correspondence is to bring this subset of children to the attention of the wider paediatric community and to optimize early recognition and management

\section{REFERENCE}

01. "Multisystem Inflammatory Syndrome in Children (MIS-C) Associated with Coronavirus Disease 2019 (COVID19)". emergency.cdc.gov. Centers for Disease Control and Prevention. 15 May 2020. Retrieved 15 May 2020.

02. Multisystem inflammatory syndrome in children and adolescents with COVID19: Scientific brief". www.who.int. World Health Organization. Archived from the original on 15 May 2020.

03. Riphagen S, Gomez X, GonzalezMartinez C, Wilkinson N, Theocharis P. Hyperinflammatory shock in children during COVID-19 pandemic. Lancet. 2020. Epub 2020/05/11.

04. Wei M, Yuan J, Liu Y, Fu T, Yu X, Zhang ZJ. Novel Coronavirus Infection in Hospitalized Infants Under 1 Year of Age in China. JAMA. 2020. Epub 2020/02/15

05. Lu X, Zhang L, Du H, Zhang J, Li YY, Qu J, et al. SARS-CoV-2 Infection in Children. N Engl J Med. 2020;382(17):1663-5. Epub 2020/03/19 